\title{
MT1-MMP-Mediated Cleavage of Decorin in Corneal Angiogenesis
}

\author{
Tatsuya Mimura ${ }^{a}$ Kyu Yeon Han ${ }^{a}$ Tatsuya Onguchi ${ }^{a} \quad$ Jin-Hong Chang ${ }^{a}$ b \\ Tae-im Kim $^{\text {b }}$ Takashi Kojimaa,b Zhongjun Zhou ${ }^{c}$ Dimitri T. Azar ${ }^{\text {a, b }}$ \\ a Department of Ophthalmology and Visual Sciences, University of Illinois at Chicago, Chicago, III., and \\ ${ }^{b}$ Department of Ophthalmology, Massachusetts Eye and Ear Infirmary, Schepens Eye Research Institute, \\ Harvard Medical School, Boston, Mass., USA; 'Department of Biochemistry, University of Hong Kong, \\ Hong Kong, SAR, China
}

\section{Key Words}

Corneal neovascularization - Angiogenesis ·

Metalloproteinase $\cdot$ MMP-14 $\cdot$ Membrane type 1-matrix

metalloproteinase $\cdot$ Decorin $\cdot$ Extracellular matrix wild-type corneal cell lysates, and an MT1-MMP knockin restores decorin processing in vitro. Conclusion: The proangiogenic role of MT1-MMP in the cornea may be mediated, in part, by facilitated cleavage of corneal decorin.

Copyright $\odot 2009$ S. Karger AG, Basel

\section{Introduction}

Angiogenesis plays a critical role in a variety of pathological conditions such as tumor growth and metastasis, diabetic retinopathy, and wound healing $[1,2]$. Identification of the factors that contribute to the physiology of angiogenic pathways and their diseases is the subject of extensive research. Several angiogenic factors, such as vascular endothelial growth factors (VEGFs), fibroblast growth factors (FGFs), angiopoietin-2, and platelet-derived growth factor, are implicated in the activation of endothelial cells [3]. Similarly, the extracellular matrix (ECM) and the matrix metalloproteinases (MMPs) also may contribute to angiogenesis and vascular development $[4,5]$.

MMPs, a family of $\mathrm{Zn}^{2+}$-dependent enzymes, are produced as latent proenzymes that may be inhibited by specific tissue inhibitors of metalloproteinases [6-9]. The MMPs are classified into two subfamilies according to

\section{KARGER}

(C) 2009 S. Karger AG, Basel

Fax +4161306 1234 E-Mail karger@karger.ch www.karger.com www.karger.com/jvr
Dr. Dimitri T. Azar

Department of Ophthalmology and Visual Sciences

University of Illinois at Chicago

1855 West Taylor Street, Chicago, IL 60612 (USA)

Tel. +1 312966 6590, Fax +1 312966 7770, E-Mail dazar@uic.edu 
structure: membrane-type (MT-MMP) and secreted MMPs [10]. Of the six MT-MMPs described thus far, MT1-MMP possesses broad-spectrum activity and cleaves various ECM components such as type I and II collagens, fibronectin, vitronectin, laminin, fibrin, and proteoglycan [11-14].

Recent evidence suggests that MT1-MMP may play an important role in cellular invasion by disrupting ECM barriers [15-18]. Furthermore, MT1-MMP participates in the proteolytic degradation of ECM components such as the basement membrane or interstitial stroma associated with angiogenesis $[19,20]$. For example, Li et al. [21] found that MT1-MMP degrades lumican and abrogates lumican-mediated suppression of tumor cell colony formation in soft agar. Abd El-Aziz et al. [22] demonstrated that MT1-MMP contributes to tumor cell proliferation through the cleavage of growth differentiation factor 15 (GDF15), a transforming growth factor- $\beta$ superfamily member. Endothelial cells from lungs of 1-week-old MMP-14 knockout $\left(\mathrm{MMP}^{-/-} 4^{-}\right.$) mice show reduced migration and limited ability to form three-dimensional structures on Matrigel [17, 18, 23-25].

Corneal lumican, decorin, and keratocan are involved in corneal morphogenesis that modulate collagen fibrillogenesis [26]. Decorin belongs to a family of structurally related proteoglycans, grouped as the small leucine-rich proteoglycans, and is a normal constituent of the corneal stroma [27]. Decorin has been shown to inhibit vascular endothelial cell migration and tube formation when endothelial cells are grown on decorin-coated surfaces [28]. Sulochana et al. [29] demonstrated that purified decorin and the leucine-rich repeat 5 (LRR5) of decorin core protein function as angiogenesis inhibitors by inhibiting both VEGF- and basic FGF (bFGF)-induced angiogenesis. Decorin binds to insulin-like growth factor I (IGF-I) receptor in a corneal inflammatory angiogenesis model [30], and IGF-I receptor is upregulated in decorin-deficient mice [31]. The addition of recombinant decorin to wild-type (WT) tumor cells caused $80-95 \%$ suppression of VEGF mRNA and protein, and resulted in decreased in vivo tumor growth [32].

Previous in situ hybridization studies demonstrated the expression of MT1-MMP in corneal keratocytes and epithelial cells in unwounded and keratectomy-wounded corneas [33]. We also demonstrated that MT1-MMP regulates angiogenesis in part by cleaving type XVIII collagen in the cornea [33]. A major component of the corneal stroma is decorin, which is a potent inhibitor of angiogenesis. In this study, we investigated the effects of MT1MMP on decorin processing and degradation, and dem- onstrated that the degradation of decorin by MT1-MMP, either directly or indirectly, results in a proangiogenic effect in the cornea.

\section{Materials and Methods}

Animals and Antisera

All animal studies were conducted in accordance with the Animal Care and Use Committee guidelines of the Massachusetts Eye and Ear Infirmary and the Association for Research in Vision and Ophthalmology Statement for the Use of Animals in Ophthalmic and Vision Research. C57BL/6 WT mice were used for in vivo experiments. Rabbit polyclonal anti-MT1-MMP antisera preparations (designated C14) that recognize the cytoplasmic domain (QRSLLDKV) of MT1-MMP were generated in-house according to established protocols. MT1-MMP knockout mice were kindly provided by Dr. Zhou and Dr. Tryggvason (Division of Matrix Biology, Department of Medical Biochemistry and Biophysics, Karolinska Institutet, Stockholm, Sweden).

\section{Corneal Micropocket Procedure}

Mouse corneal micropocket procedures were performed as previously described [34, 35]. Mice were anesthetized using a combination of ketamine and xylazine injections. Supplemental proparacaine eye drops were used for local anesthesia. Corneal micropockets were created using a modified von Graefe knife in WT mice. Hydron pellets $(0.4 \times 0.4 \mathrm{~mm})$ containing $120 \mathrm{ng}$ of human recombinant bFGF (R\&D Systems, Minneapolis, Minn., USA) were implanted into the corneal pockets. Ofloxacin eye drops were instilled after surgery. The eyes were photographed by slit lamp microscopy (Nikon, Tokyo, Japan) and enucleated for immunohistochemistry and Western blot analysis on postoperative day 7 .

\section{Western Blotting}

Unwounded WT mouse corneas and corneas that were implanted with pellets were excised on day 7. The cells were lysed in a buffer containing $150 \mathrm{mM} \mathrm{NaCl}, 0.25 \%$ deoxycholic acid, $1 \%$ Nonidet $\mathrm{P}-40$, and $50 \mathrm{~mm}$ Tris- $\mathrm{HCl}$ ( $\mathrm{pH}$ 7.6), mixed with a sample buffer containing $\beta$-mertcaptoethanol, boiled for $2 \mathrm{~min}$, and subjected to Western blot analysis. Sodium dodecyl sulfate-polyacrylamide gel electrophoresis (SDS-PAGE) was conducted using 4-20\% Tris-glycine gradient gels (Invitrogen, Carlsbad, Calif., USA). After SDS-PAGE, proteins were transferred electrophoretically to a hydrophobic polyvinylidene difluoride membrane (Immobilon-P; Millipore, Bedford, Mass., USA). The membrane was incubated at room temperature (RT) for $1 \mathrm{~h}$ in $1 \times$ TBST (Tris-buffered saline Tween 20) containing 3\% BSA (Sigma-Aldrich, St. Louis, Mo., USA), and then incubated at RT for $1 \mathrm{~h}$ in TBST containing primary antibody (goat antimouse decorin antibody, 1:500; R\&D Systems, or rabbit anti-MT1-MMP polyclonal antisera, 1:1,000). The membrane was then incubated at RT with shaking for 30 min in TBST containing the second antibody (horseradish peroxidase-conjugated donkey anti-goat IgG, 1:20,000; Pierce, Rockford, Ill., USA, or donkey anti-rabbit IgG, 1:20,000; Amersham Biosciences, Little Chalfont, UK). Each step was followed by extensive washing in TBST. Subsequently, the membrane was washed 3 times for $10 \mathrm{~min}$ at RT with 
$1 \times$ TBST. Antigen detection was achieved by incubation of the membrane for $1 \mathrm{~min}$ at RT with a chemiluminescent substrate (SuperSignal West Pico; Pierce) and exposure to X-Omat Blue XB-1 film (Kodak, Rochester, N.Y., USA) for $10 \mathrm{~s}$ or, if necessary, up to $30 \mathrm{~min}$.

\section{Confocal Immunohistochemistry}

Mouse corneas were embedded in OCT compound (TissueTek; Miles Laboratories, Naperville, Ill., USA) at $-20^{\circ} \mathrm{C}$. Frozen sections were cut at a thickness of $8 \mu \mathrm{m}$, placed on microscope slides, air dried, and then fixed in $4 \%$ paraformaldehyde for 5 min. After blocking with 3\% BSA in PBS, sections were incubated at RT for $2 \mathrm{~h}$ with specific primary antibodies diluted in BSA/TBST. Either goat anti-mouse decorin antibody (1:100) or rabbit anti-MT1-MMP (C14) antisera (1:100) were used for staining. After washing with PBS, sections were incubated at RT for 30 min with FITC-conjugated anti-goat or anti-rabbit IgG secondary antibodies (1:400; both from Jackson ImmunoResearch Laboratories, West Grove, Pa., USA). Stained sections were observed by confocal microscopy (TCS 4D; Leica, Heidelberg, Germany). Propidium iodide (Vector Laboratories, Burlingame, Calif., USA) was used for nuclear staining.

\section{Aortic Ring Assays}

Aortic ring assays were performed as described [36]. Briefly, aortas were obtained from WT mice, and fatty tissues around the aorta were removed carefully under a surgical microscope. A 1$\mathrm{mm}$-long aortic ring was cut and rinsed in five consecutive washes of EBM (Cambrex, Walkersville, Md., USA). Wells of 48-well plates were coated with $150 \mu \mathrm{l}$ of rat tail type I collagen (BD Biosciences, Bedford, Mass., USA). After gelling at $37^{\circ} \mathrm{C}$ for $30 \mathrm{~min}$, an aortic ring was placed on its side on top of the gel and sealed in place with an overlay of $100 \mu \mathrm{l}$ of collagen. One hour later, 300 $\mu \mathrm{l}$ of EBM containing $20 \mathrm{ng} / \mathrm{ml}$ purified decorin (Sigma-Aldrich) and/or $20 \mathrm{ng} / \mathrm{ml}$ recombinant human MT1-MMP (Calbiochem, Gibbstown, N.J., USA) was added to each well. On days 6 and 9, the gels were photographed with a phase contrast microscope equipped with a digital spot camera (Micro Video Instruments, Avon, Mass., USA). Tube formation was determined by counting the number of microvessels and analyzing according to reported criteria [36].

\section{Immortalized Corneal Keratocyte Cell Lines}

Immortalized mouse corneal cell lines from WT mice were generated as described previously [37] and immunostained with antivimentin antibodies. Briefly, corneal keratocytes were isolated by incubating MT1-MMP knockout mouse corneas, immortalized with SV40T large T antigen and subcloned via serial dilutions. To generate MT1-MMP knockin cell lines, the MT1-MMP cDNA construct was subcloned into the $\mathrm{pFB}$ vector under CMV promoter followed with internal ribosomal entry sequence (IRES) and EGFP coding sequence. Recombinant viruses were generated by transfection of $293 \mathrm{~T}$ cells with the pFB vector encoding MT1MMP or an empty control vector using Effectene ${ }^{\mathrm{TM}}$ transfection reagent (Qiagen, Valencia, Calif., USA). MT1-MMP containing virus was generated and used to infect MT1-MMP knockout keratocyte cell lines. The medium obtained from the second and third days after transfection, which has higher virus expression, was collected and used for the infection of corneal cell lines. The corneal keratocyte cell lines from WT or MT1-MMP KO mice were infected with control or MT1-MMP virus, and the cells were analyzed by flow cytometry on the Coulter EPICS XL-MCL flow cytometer (Coulter Electronics, Miami, Fla., USA). Stained cells were sorted using a Coulter ELITE cell sorter into EGFP-positive and EGFP-negative cells. The stability of the expression of EGFP was monitored by flow cytometry.

EGFP-positive cells were then plated at a concentration of 10,000 cells $/ \mathrm{ml}$ in DMEM, supplemented with $10 \%$ heat-inactivated $\mathrm{FCS}$ at $37^{\circ} \mathrm{C}$ in $5 \% \mathrm{CO}_{2}$. DMEM containing $0.5 \%$ FCS was used for starvation conditions. When the cells reached $80 \%$ confluence, the cells were kept in the serum-free medium. After $24 \mathrm{~h}$, cells were collected and lysed in $1 \mathrm{ml}$ of RIPA buffer [50 mM Tris/ $\mathrm{HCl}(\mathrm{pH} 7.5) / 150 \mathrm{mM} \mathrm{NaCl} / 1 \%$ (v/v) Nonidet P-40/0.25\% (w/v) sodium deoxycholate] and incubated at $4^{\circ} \mathrm{C}$ for $30 \mathrm{~min}$. The purpose of the 30 -min incubation was to solubilize the cellular protein.

\section{Cell Lysate-Mediated Decorin Cleavage Assay}

All cleavage assays were carried out in a volume of $50 \mu$ l. One hundred nanograms of decorin was incubated in reaction buffer [60 mM Tris- $\mathrm{HCl}$ (pH 6.5), $150 \mathrm{mM} \mathrm{NaCl}, 25 \mu \mathrm{M} \mathrm{ZnSO}$, and 25 $\mu \mathrm{M} \mathrm{CaCl}_{2}$ ] with corneal keratocyte cell lysates obtained from cultured WT, MT1-MMP KO, and KO/MT1-MMP mouse keratocytes. RIPA buffer was used as the control. The degradation pattern of decorin was analyzed by gel electrophoresis and Western blot using antidecorin antibodies (R\&D Systems).

\section{Data Analysis}

Statistical analyses were performed using the SPSS program (Chicago, Ill., USA). Results were expressed as mean \pm SEM. Data was analyzed using analysis of variance (ANOVA), and a value of $\mathrm{p}<0.05$ was considered statistically significant.

\section{Results}

\section{Expression of MT1-MMP in bFGF-Induced Corneal Neovascularization}

To examine the expression of MT1-MMP, the C14 anti-MT1-MMP antisera, which recognizes the cytoplasmic domain of MT1-MMP, was used to stain normal and vascularized mouse corneas. Seven days following bFGF pellet implantation into mouse corneas to induce neovascularization, immunohistochemistry revealed positive reactivity against MT1-MMP in the stroma (fig. 1). MT1-MMP expression was detected in bFGF-treated corneas (fig. 1b). The specificity of the C14 MT1-MMP antisera was verified by preabsorption of cognate peptide antigen, and little to no detectable MT1-MMP immunostaining was present in the vascularized cornea (fig. 1e). Western blot analysis using the C14 antisera revealed strong bands in the corneas that were $58 \mathrm{kDa}$ in size (fig. 1h). 

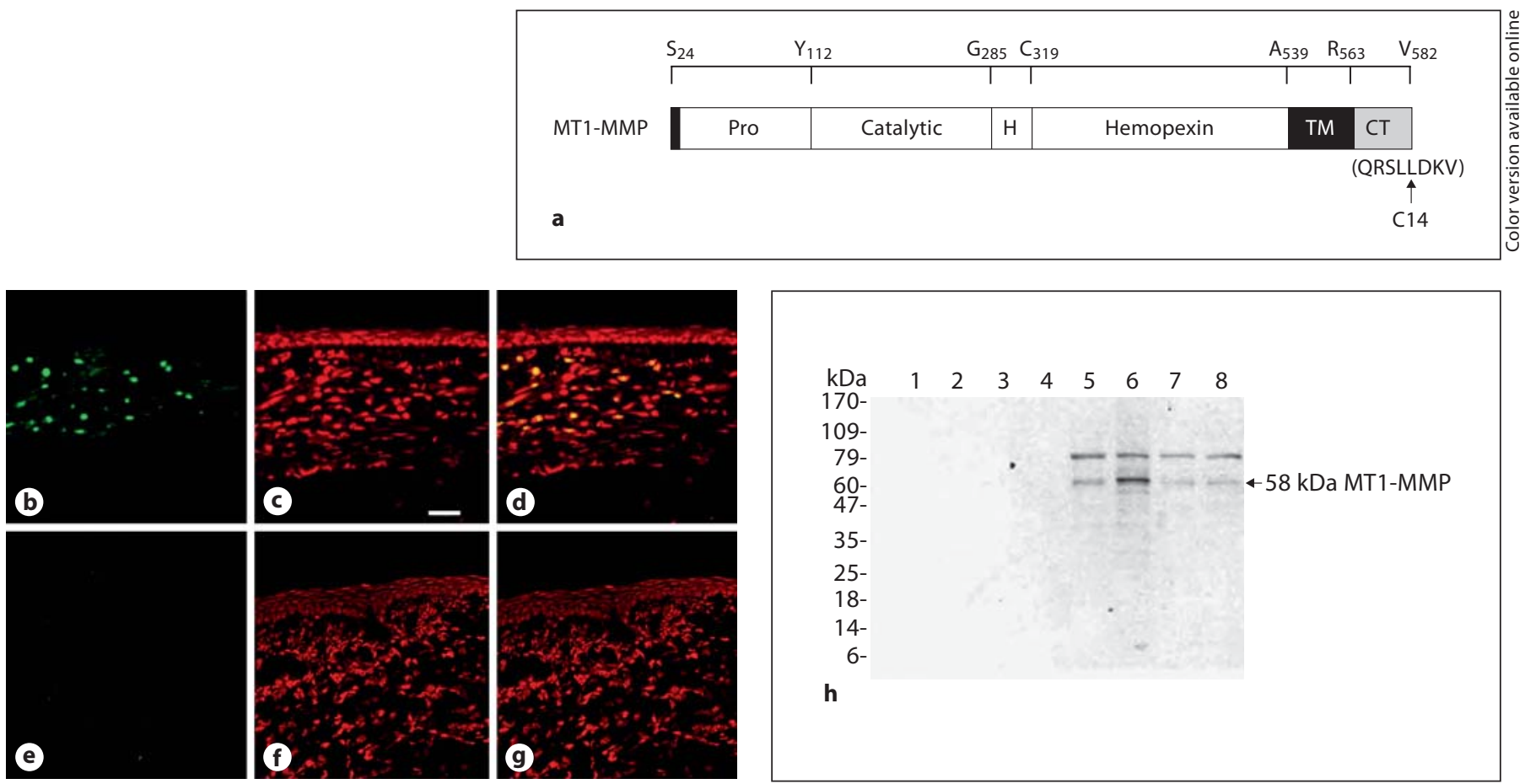

Fig. 1. Enhanced MT1-MMP expression in vascularized cornea. a Rabbit polyclonal antiserum, which recognizes the cytoplasmic domain (C14) of MT1-MMP, was generated. The schematic diagram shows the various domains of the MT1-MMP protein (Pro = prodomain; $\mathrm{H}=$ hinge region; $\mathrm{TM}=$ transmembrane domain; CT = cytoplasmic tail). $\mathbf{b}-\mathbf{d}$ The expression of MT1-MMP was examined using immunofluorescence. The expression of MT1-MMP was examined in the mouse corneas 7 days after bFGF pellet implantation and prominent staining of MT1-MMP was

\section{Expression and Cleavage of Decorin in bFGF-Induced} Corneal Neovascularization

Because decorin is known to be a potent inhibitor of angiogenesis and is a major component of the corneal stroma, the expression of decorin was analyzed in normal and vascularized corneas using immunohistochemistry, and cleavage was examined using Western blot analysis. In the normal mouse cornea, decorin was expressed throughout the entire depth of the stroma, as expected (fig. 2a-c). However, in bFGF pellet-induced vascularized corneas, decorin was not detected around corneal vessels and pellet areas (fig. $2 \mathrm{~d}-\mathrm{f}$ ).

The degradation pattern of decorin was compared between normal and vascularized corneas using Western blot analysis (fig. 2j). Presumed decorin degradation products were noted as lower molecular weight fragments in vascularized corneas and were not detected in normal corneas (fig. 2j).

\section{Cleavage of Decorin by MT1-MMP}

To analyze the cleavage of decorin by MT1-MMP, the two proteins were incubated under various experimental conditions in vitro. Western blot analysis indicated that decorin was cleaved by MT1-MMP in the presence of $\mathrm{Zn}^{2+}$ and $\mathrm{Ca}^{2+}$, as little to no MT1-MMP-mediated cleavage of decorin was observed in the absence of $\mathrm{Zn}^{2+}$ and $\mathrm{Ca}^{2+}$ (fig. 3a). Decorin proteolysis by MT1-MMP was more efficient at $\mathrm{pH} 5.5$ and $\mathrm{pH} 6.5$ when compared to that at $\mathrm{pH}$ 7.6 (fig. 3b). The kinetics of decorin proteolytic processing by MT1-MMP occurred in a concentration- and time-dependent manner (fig. $3 \mathrm{c}$ and d, respectively). Minimal cleavage was observed after $1 \mathrm{~h}$, and complete cleavage of the substrate required almost $20 \mathrm{~h}$ (fig. 3d). GM6001 (CalBiochem, Darmstadt, Germany) inhibited MT1-MMP cleavage of recombinant decorin (fig. 3e). 

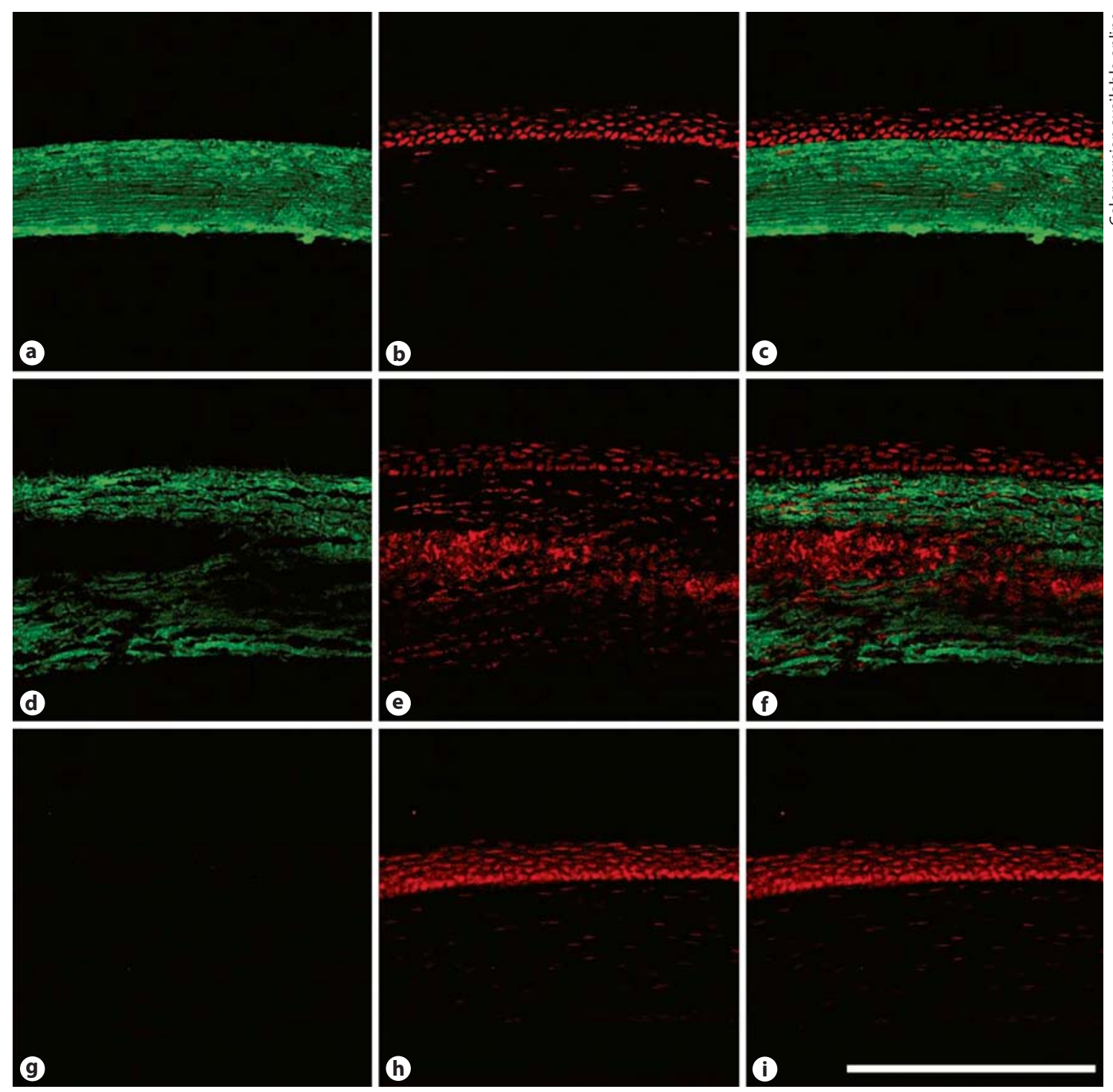

Fig. 2. Expression of decorin in vascularized corneas. bFGF pellets were implanted into mouse corneas. After 7 days, the corneas were sectioned and immunoblotted with antidecorin antibodies and the expression of decorin was examined by immunofluorescence $(\mathbf{a}-\mathbf{i})$ and Western blot analysis $(\mathbf{j})$. The first column of the immunofluorescence analysis $(\mathbf{a}, \mathbf{d}, \mathbf{g})$ shows staining of decorin, the second column $(\mathbf{b}, \mathbf{e}, \mathbf{h})$ shows propidium iodide staining, and the third column $(\mathbf{c}, \mathbf{f}, \mathbf{i})$ shows the merged image. a Decorin was expressed throughout the normal corneal stroma. d Little to no expression of decorin was detected around the pellet area in the corneal stroma. g Primary antibodies were omitted as a negative control. $\mathbf{j}$ Western blot analysis suggests that decorin cleavage into small fragments was greater in vascularized corneas $(n=4$, lanes $5-8)$ as compared to normal corneas ( $\mathrm{n}=4$, lanes $1-4)$.

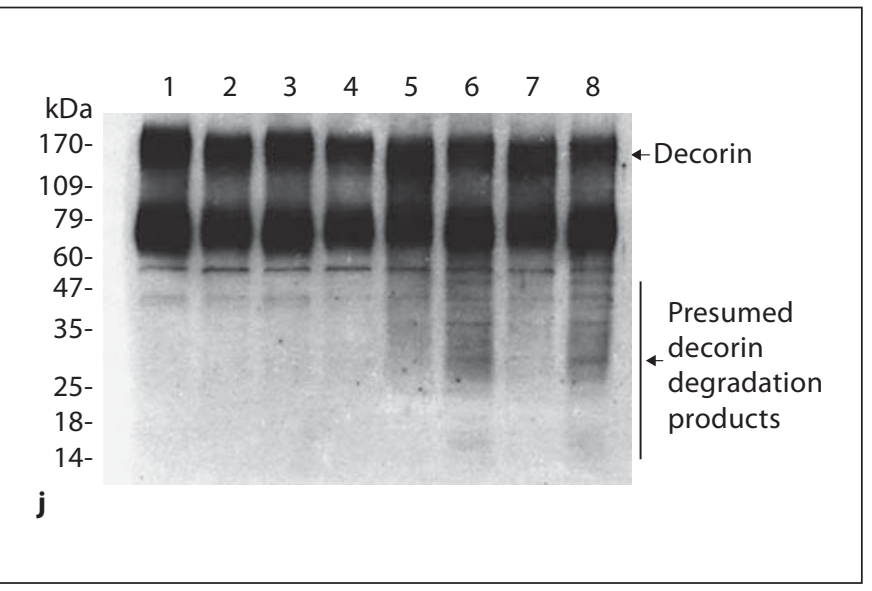




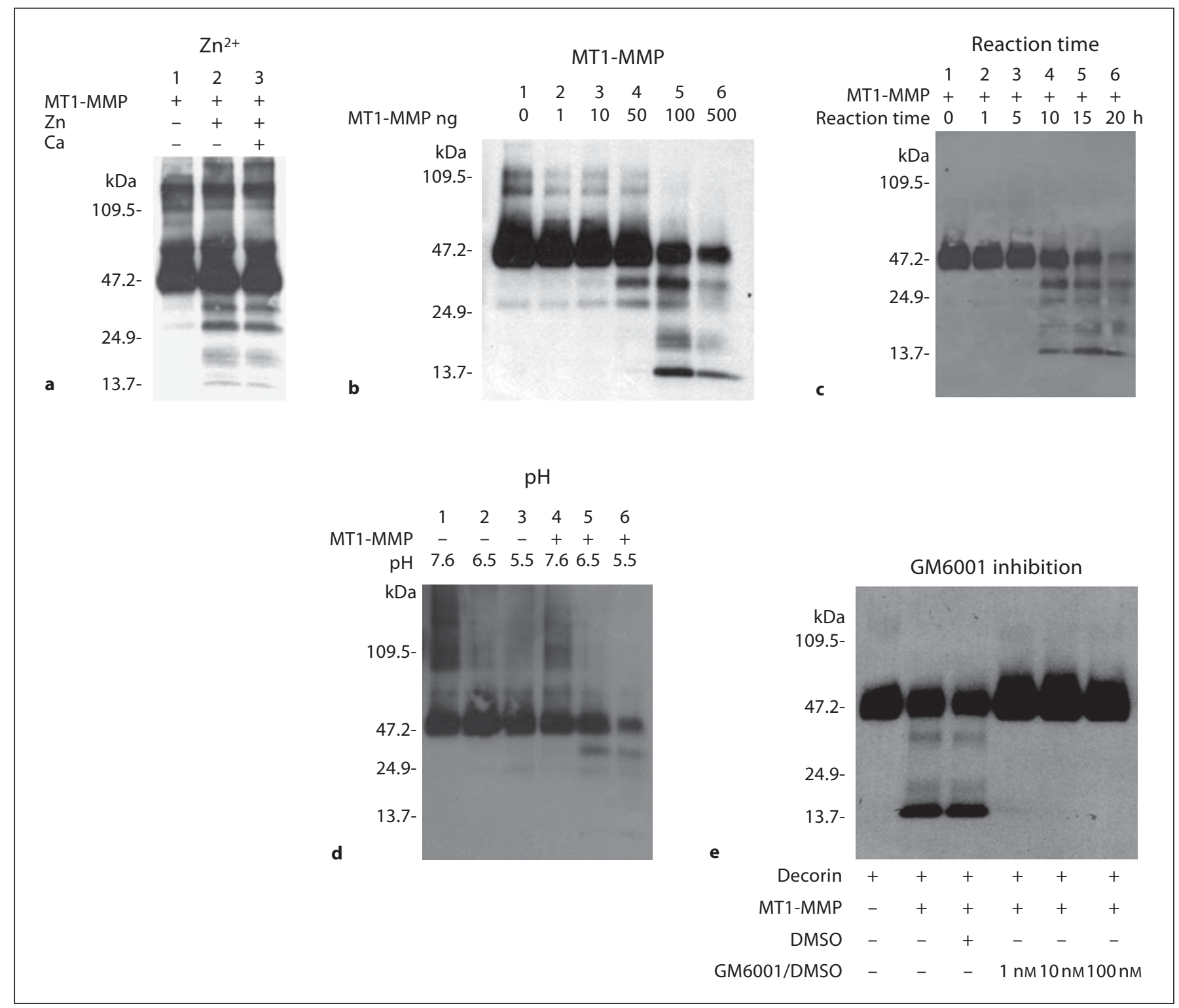

Fig. 3. In vitro cleavage of decorin by MT1-MMP. Decorin was incubated with various concentrations of human recombinant MT1-MMP at different time points (from 0 to $20 \mathrm{~h}$ ), with differential $\mathrm{Zn}^{2+}$ and $\mathrm{Ca}^{2+}$ concentrations and levels of $\mathrm{pH}$. The degradation pattern of decorin was analyzed by Western blot analysis. a Decorin was processed by MT1-MMP in vitro in the presence of $\mathrm{Zn}^{2+}$ and $\mathrm{Ca}^{2+}$. $\mathbf{b}$ The kinetics of decorin proteolytic processing by MT1-MMP is regulated by MT1-MMP concentrations and incubation time (c). Decorin proteolysis by MT1-MMP was enhanced at $\mathrm{pH} 6.5$ when compared to that of physiologic $\mathrm{pH} 7.6$ (d). GM6001 (1, 10 and $100 \mathrm{nM}$ ) inhibited MT1-MMP cleavage of recombinant decorin (e).

\section{Aortic Ring Tube Formation Inhibition by Decorin} and Reversal by the Addition of Recombinant Active MT1-MMP

To assess the proangiogenic activity of MT1-MMP in vitro in the presence of decorin, mouse aortic rings embedded in collagen gel were maintained in EBM containing MT1-MMP and/or decorin. The in vitro sprouting of microvessels in the aortic ring assay at days 6 and 9 was inhibited by media containing decorin (fig. $4 \mathrm{~b}$ and e, respectively). When MT1-MMP was added to decorin-containing media, the number of microvessel tubes was increased at days 6 and 9 (fig. $4 \mathrm{c}$ and f, respectively), suggesting that the proangiogenic effect of MT1-MMP offsets the antiangiogenic effect of decorin (fig. $4 \mathrm{~g}$ ). 


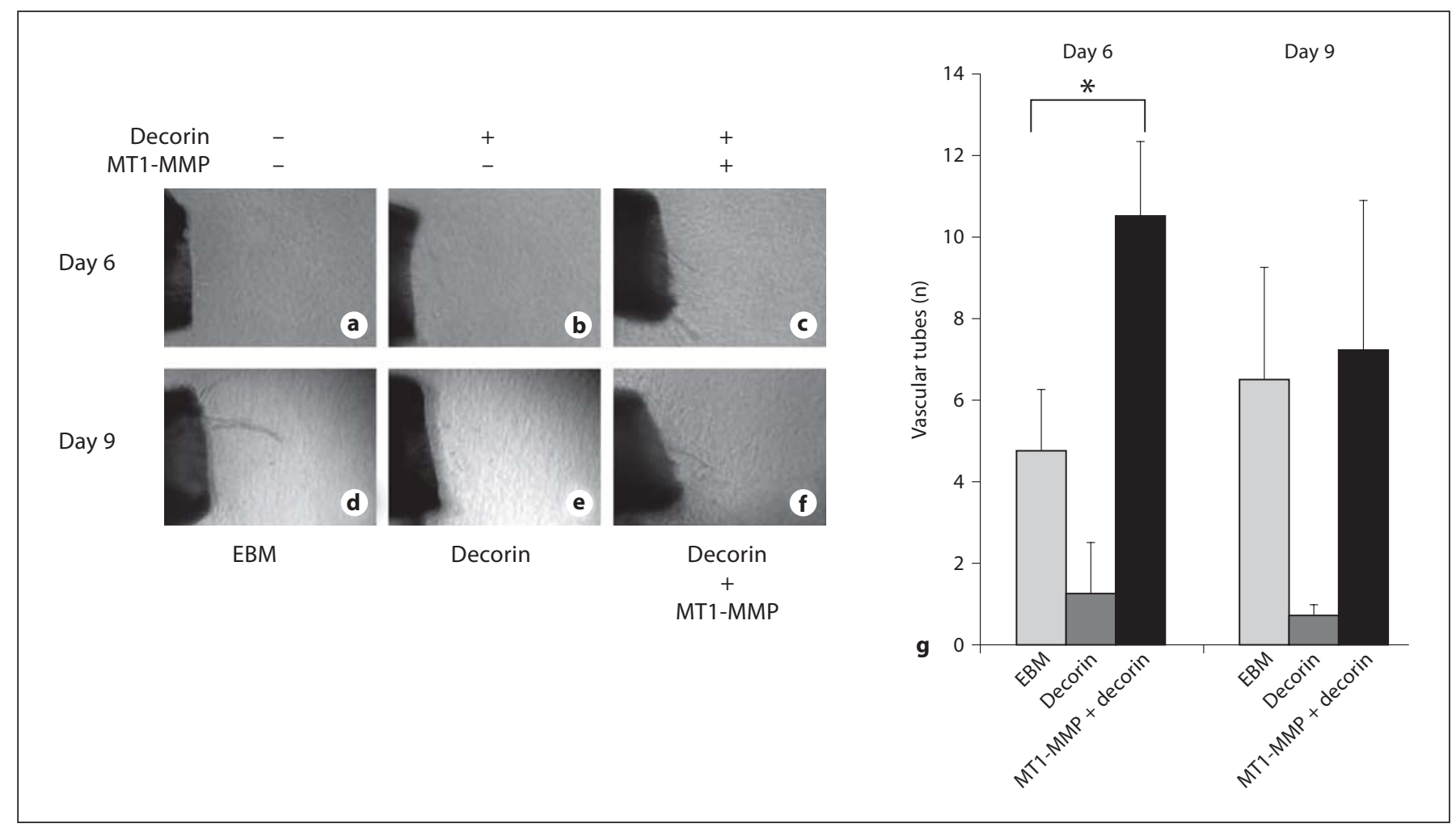

Fig. 4. Enhanced vascular tube formation in the presence of decorin and recombinant MT1-MMP in an aortic ring assay. Mouse aortas were embedded in type I collagen and then bathed in complete EBM only or complete EBM supplemented with $20 \mathrm{ng} / \mathrm{ml}$ MT1-MMP and/or $20 \mathrm{ng} / \mathrm{ml}$ decorin as indicated. Photographs were taken on days $6(\mathbf{a}-\mathbf{c})$ and $9(\mathbf{d}-\mathbf{f})$ after embedding the aortas. The numbers of microvessels were counted under the microscope at each time point. Vascular tube formation was inhibited by

\section{Cleavage of Decorin by Cell Lysates or Culture}

Medium Obtained from Cultured Keratocytes

MT1-MMP mRNA was determined by real-time PCR in WT-Ker, KO-Ker, and KO-Ker/MT1-MMP cell lines (fig. 5a). To further examine decorin proteolysis, the MT1-MMP-mediated cleavage of decorin was assayed in the presence of cell lysates from mouse corneal keratocytes. The addition of cell lysates from WT keratocytes (fig. 5b) enhanced the cleavage of decorin to generate decorin degradation products. Reduced amounts of decorin degradation fragments $(25-35 \mathrm{kDa})$ were observed following the addition of cell lysates from cultured MT1MMP KO keratocytes (fig. 5b). Decorin degradation was reestablished in the presence of lysates from MT1-MMP knockin keratocytes (fig. 5b). These results suggest that MT1-MMP derived from WT and knockin keratocytes may contribute to decorin degradation. decorin (b, e). Despite the presence of decorin, the addition of MT1-MMP diminished the inhibitory effects on microvessel tube formation mediated by decorin (c, f). Microvessels were calculated as mean $\pm \operatorname{SEM}(\mathbf{g})$. ANOVA showed statistically significant differences between decorin and decorin + MT1-MMPtreated groups as well as the EBM control group on day $6\left({ }^{*} \mathrm{p}<\right.$ $0.05)$, but not day 9 .

\section{Discussion}

Our current findings support the hypothesis that the proangiogenic effect of corneal MT1-MMP may be mediated, in part, by the cleavage of an antiangiogenic factor, decorin. Other possible proangiogenic mechanisms of MT1-MMP include: (1) the production of angiogenic factors, such as VEGF, (2) the degradation of antiangiogenic factors in addition to decorin, and/or (3) the breakdown of ECM components to facilitate vascular endothelial cell migration. These mechanisms could occur either directly or via MMP-2 activation, as we previously reported that corneal angiogenesis is diminished upon bFGF stimulation in mice deficient in MMP-2, a substrate of MT1-MMP [35]. Alternatively, additional substrates of MT1-MMP may exist to play a role. Tam et al. [38] used membrane proteomics to characterize several 


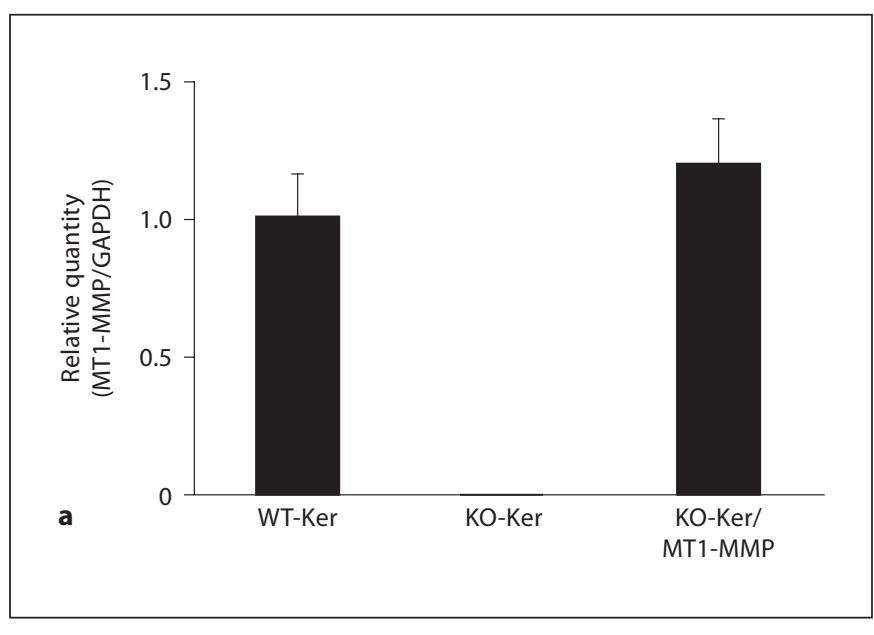

Fig. 5. Decorin processed by cultured keratocyte cell lysates. Immortalized corneal cells from WT and MT1-MMP KO mice were subcloned to generate corneal keratocyte cells. MT1-MMP KO cells were transfected with MT1-MMP (KO/MT1-MMP). a Realtime PCR showed no MT1-MMP mRNA detection in KO-Ker cells using primer from exon 4. KO-Ker/MT1-MMP restored MT1-MMP mRNA expression. b Cleavage of decorin was ana-

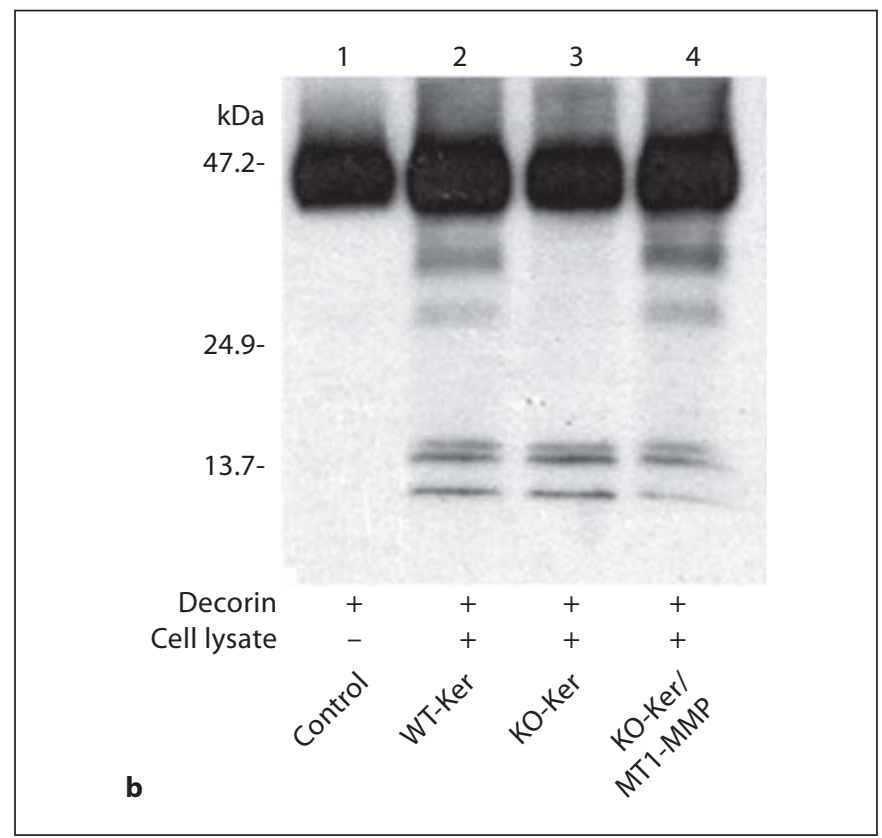

lyzed in the presence of cell lysates using Western blot analysis. Decorin was incubated with $20 \mu \mathrm{l}$ of cell lysates obtained from the various cultured mouse keratocytes for $24 \mathrm{~h}$. The presence of the 25 - to $35-\mathrm{kDa}$ bands shows that decorin was cleaved more efficiently by the WT (lane 2) and KO/MT1-MMP cell lysates (lane 4) than by the KO cell lysate (lane 3 ). Buffer in the absence of cell lysate was used as a control (lane 1). potential MT1-MMP substrates. These substrates include ECM proteins and signaling molecules such as fibronectin, laminin, type I collagen, MMP-2, and MMP-13, TNF- $\alpha$ and connective tissue growth factor [38]. Additionally, Abd El-Aziz et al. [22] have shown that MT1MMP contributes to tumor cell proliferation through the cleavage of GDF15, which downregulates cell proliferation.

Although mice deficient in other MMPs show minimal apparent phenotypic changes, MT1-MMP-deficient mice exhibit an extremely disfigured phenotype due to inadequate collagen turnover and marked deceleration of postnatal growth, which leads to death at approximately 3 weeks of age. Additionally, these mice display a deficiency in the ability to induce corneal neovascularization after bFGF pellet implantation [39]. Due to the lack of appropriate anti-mouse MT1-MMP antibodies, characterization of the temporal and spatial expression patterns of MT1-MMP, and assessment of MT1-MMP involvement during bFGF pellet implantation was difficult. In this study, we successfully generated polyclonal antisera to a discrete region of mouse MT1-MMP, specifically to the cytoplasmic (C14) domain. It is not clear whether the $58-\mathrm{kDa}$ band recognized by the antisera represents truncated or active MT1-MMP. However, it is likely that the 79-kDa band represents full length Pro-MT1-MMP. Additional studies are needed to further clarify these questions.

The mouse corneal model is well suited for characterizing proangiogenic and antiangiogenic responses, given that the mouse cornea contains several well-characterized ECM molecules with known antiangiogenic properties, including collagen XVIII, collagen IV, decorin, and thrombospondin. We have shown previously that collagen XVIII is proteolytically processed by MT1-MMP. Our current findings demonstrate that MT1-MMP is involved in the cleavage of decorin, a previously uncharacterized substrate, and this process may regulate corneal neovascularization. Increasing evidence suggests that decorin possesses antiangiogenic properties. Jarvelainen et al. [40] found that decorin influenced new vessel formation in the cutaneous wound-healing model in deco- 
rin-deficient mice. Additionally, Grant et al. [32] demonstrated that decorin suppresses tumor cell-mediated angiogenesis byinhibitingtumor-derived VEGF production. Furthermore, Davies Cde et al. [28] found that decorin alone, or in combination with thrombospondin-1, inhibited angiogenesis in vitro. Lastly, Nelimarkka et al. [41] reported that decorin might play a role in inflammationassociated angiogenesis, given that capillary endothelial cells in pyogenic granuloma and granulation tissue of healing dermal wounds produced decorin. However, other reports suggest that decorin promotes rather than inhibits angiogenesis. Schonherr et al. [31] showed that a decorin deficiency leads to impaired angiogenesis in injured mouse corneas in a chemical cauterization model. Importantly, the studies suggesting that decorin is either an inhibitor or a stimulator of angiogenesis are not necessarily contradictory, rather they indicate the molecular complexity of angiogenesis under differential physiologic and pathologic conditions.

In this report, we demonstrate bFGF-induced MT1MMP expression and diminished decorin expression around bFGF-pellet-implanted areas in vascularized mouse corneas. Additionally, we show that MT1-MMP cleaves decorin in vitro and that cell lysates from MT1MMP-deficient keratocytes have lost their decorin-processing activity. Lastly, we find that purified decorin inhibits vascular tube formation in an aortic ring assay and that the addition of recombinant active MT1-MMP reverses the decorin-mediated inhibition. Although our data suggest a role for decorin in MT1-MMP-mediated angiogenesis pathways, other antiangiogenic factors also may be involved in MT1-MMP-mediated corneal neovascularization, as previously mentioned. Based on the important role of MT1-MMP and decorin in angiogenesis, an understanding of the mechanism of MT1-MMP/ decorin signaling and regulation in angiogenesis may provide the basis for therapeutic interventions for the treatment of corneal neovascularization, and possibly other angiogenesis-related disorders.

\section{Acknowledgments}

This study was funded by grants from the National Institutes of Health EY10101 (D.T.A.), P30EY001792 (D.T.A.), and EY14048 (J.-H.C.).

\section{References}

1 Folkman J: Angiogenesis. Annu Rev Med 2006;57:1-18.

2 Azar DT: Corneal angiogenic privilege: angiogenic and antiangiogenic factors in corneal avascularity, vasculogenesis, and wound healing (an American Ophthalmological Society thesis). Trans Am Ophthalmol Soc 2006; 104:264-302.

- 3 Chang JH, Gabison EE, Kato T, Azar DT: Corneal neovascularization. Curr Opin Ophthalmol 2001;12:242-249.

4 Raffetto JD, Khalil RA: Matrix metalloproteinases and their inhibitors in vascular remodeling and vascular disease. Biochem Pharmacol 2008;75:346-359.

5 Rundhaug JE: Matrix metalloproteinases and angiogenesis. J Cell Mol Med 2005;9: 267-285.

-6 Werb Z, Chin JR: Extracellular matrix remodeling during morphogenesis. Ann NY Acad Sci 1998;857:110-118.

7 Nagase H: Activation mechanisms of matrix metalloproteinases. Biol Chem 1997;378: 151-160.

8 Nagase H, Visse R, Murphy G: Structure and function of matrix metalloproteinases and TIMPs. Cardiovasc Res 2006;69:562-573.
9 Sato H, Kinoshita T, Takino T, Nakayama K, Seiki M: Activation of a recombinant membrane type 1-matrix metalloproteinase (MT1-MMP) by furin and its interaction with tissue inhibitor of metalloproteinases (TIMP)-2. FEBS Lett 1996;393:101-104.

10 Sato H, Seiki M: Membrane-type matrix metalloproteinases (MT-MMPS) in tumor metastasis. J Biochem (Tokyo) 1996;119:209215.

-11 d'Ortho MP, Will H, Atkinson S, Butler G Messent A, Gavrilovic J, Smith B, Timpl R, Zardi L, Murphy G: Membrane-type matrix metalloproteinases 1 and 2 exhibit broadspectrum proteolytic capacities comparable to many matrix metalloproteinases. Eur J Biochem 1997;250:751-757.

12 Ohuchi E, Imai K, Fujii Y, Sato H, Seiki M, Okada Y: Membrane type 1 matrix metalloproteinase digests interstitial collagens and other extracellular matrix macromolecules. J Biol Chem 1997;272:2446-2451.

13 Koshikawa N, Schenk S, Moeckel G, Sharabi A, Miyazaki K, Gardner H, Zent R, Quaranta V: Proteolytic processing of laminin-5 by MT1-MMP in tissues and its effects on epithelial cell morphology. FASEB J 2004; 18: 364-366.
14 Hiraoka N, Allen E, Apel IJ, Gyetko MR, Weiss SJ: Matrix metalloproteinases regulate neovascularization by acting as pericellular fibrinolysins. Cell 1998;95:365-377.

15 Wolf K, Wu YI, Liu Y, Geiger J, Tam E, Overall C, Stack MS, Friedl P: Multi-step pericellular proteolysis controls the transition from individual to collective cancer cell invasion. Nat Cell Biol 2007;9:893-904.

16 Ries C, Egea V, Karow M, Kolb H, Jochum M, Neth P: MMP-2, MT1-MMP, and TIMP-2 are essential for the invasive capacity of human mesenchymal stem cells: differential regulation by inflammatory cytokines. Blood 2007;109:4055-4063.

17 Filippov S, Koenig GC, Chun TH, Hotary KB, Ota I, Bugge TH, Roberts JD, Fay WP, Birkedal-Hansen H, Holmbeck K, Sabeh F, Allen ED, Weiss SJ: MT1-matrix metalloproteinase directs arterial wall invasion and neointima formation by vascular smooth muscle cells. J Exp Med 2005;202:663-671.

18 Hotary KB, Allen ED, Brooks PC, Datta NS, Long MW, Weiss SJ: Membrane type I matrix metalloproteinase usurps tumor growth control imposed by the three-dimensional extracellular matrix. Cell 2003;114:33-45.

19 Nagase H: Cell surface activation of progelatinase A (proMMP-2) and cell migration. Cell Res 1998;8:179-186. 
-20 Seiki M, Mori H, Kajita M, Uekita T, Itoh Y: Membrane-type 1 matrix metalloproteinase and cell migration. Biochem Soc Symp 2003; 70:253-262.

-21 Li Y, Aoki T, Mori Y, Ahmad M, Miyamori $\mathrm{H}$, Takino T, Sato H: Cleavage of lumican by membrane-type matrix metalloproteinase-1 abrogates this proteoglycan-mediated suppression of tumor cell colony formation in soft agar. Cancer Res 2004;64:7058-7064.

-22 Abd El-Aziz SH, Endo Y, Miyamaori H, Takino T, Sato H: Cleavage of growth differentiation factor 15 (GDF15) by membrane type 1-matrix metalloproteinase abrogates GDF15-mediated suppression of tumor cell growth. Cancer Sci 2007;98:1330-1335.

-23 Hotary KB, Yana I, Sabeh F, Li XY, Holmbeck K, Birkedal-Hansen H, Allen ED, Hiraoka N, Weiss SJ: Matrix metalloproteinases (MMPS) regulate fibrin-invasive activity via MT1-MMP-dependent and -independent processes. J Exp Med 2002;195:295308.

-24 Cao J, Chiarelli C, Kozarekar P, Adler HL: Membrane type 1-matrix metalloproteinase promotes human prostate cancer invasion and metastasis. Thromb Haemost 2005;93: 770-778.

-25 Oblander SA, Zhou Z, Galvez BG, Starcher B, Shannon JM, Durbeej M, Arroyo AG, Tryggvason K, Apte SS: Distinctive functions of membrane type 1 matrix-metalloprotease (MT1-MMP or MMP-14) in lung and submandibular gland development are independent of its role in pro-MMP-2 activation. Dev Biol 2005;277:255-269.
26 McEwan PA, Scott PG, Bishop PN, Bella J: Structural correlations in the family of small leucine-rich repeat proteins and proteoglycans. J Struct Biol 2006;155:294-305.

27 Hocking AM, Shinomura T, McQuillan DJ: Leucine-rich repeat glycoproteins of the extracellular matrix. Matrix Biol 1998;17:119.

28 Davies Cde L, Melder RJ, Munn LL, MoutaCarreira C, Jain RK, Boucher Y: Decorin inhibits endothelial migration and tube-like structure formation: role of thrombospondin-1. Microvasc Res 2001;62:26-42.

29 Sulochana KN, Fan H, Jois S, Subramanian V, Sun F, Kini RM, Ge R: Peptides derived from human decorin leucine-rich repeat 5 inhibit angiogenesis. J Biol Chem 2005;280 27935-27948

-30 Schonherr E, Sunderkotter C, Iozzo RV, Schaefer L: Decorin, a novel player in the insulin-like growth factor system. J Biol Chem 2005;280:15767-15772.

-31 Schonherr E, Sunderkotter C, Schaefer L, Thanos S, Grassel S, Oldberg A, Iozzo RV, Young MF, Kresse H: Decorin deficiency leads to impaired angiogenesis in injured mouse cornea. J Vasc Res 2004;41:499-508.

32 Grant DS, Yenisey C, Rose RW, Tootell M, Santra M, Iozzo RV: Decorin suppresses tumor cell-mediated angiogenesis. Oncogene 2002;21:4765-4777.

33 Ye HQ, Maeda M, Yu FS, Azar DT: Differential expression of MT1-MMP (MMP-14) and collagenase III (MMP-13) genes in normal and wounded rat corneas. Invest Ophthalmol Vis Sci 2000;41:2894-2899.

>34 Kenyon BM, Voest EE, Chen CC, Flynn E, Folkman J, D'Amato RJ: A model of angiogenesis in the mouse cornea. Invest Ophthalmol Vis Sci 1996;37:1625-1632.
35 Kato T, Kure T, Chang JH, Gabison EE, Itoh T, Itohara S, Azar DT: Diminished corneal angiogenesis in gelatinase A-deficient mice. FEBS Lett 2001;508:187-190.

36 Kojima T, Chang JH, Azar DT: Proangiogenic role of ephrinB1/EphB1 in basic fibroblast growth factor-induced corneal angiogenesis. Am J Pathol 2007;170:764-773.

37 Beales MP, Funderburgh JL, Jester JV, Hassell JR: Proteoglycan synthesis by bovine keratocytes and corneal fibroblasts: maintenance of the keratocyte phenotype in culture. Invest Ophthalmol Vis Sci 1999;40: 1658-1663.

38 Tam EM, Morrison CJ, Wu YI, Stack MS, OverallCM:Membrane protease proteomics: isotope-coded affinity tag MS identification of undescribed MT1-matrix metalloproteinase substrates. Proc Natl Acad Sci USA 2004; 101:6917-6922.

-39 Zhou Z, Apte SS, Soininen R, Cao R, Baaklini GY, Rauser RW, Wang J, Cao Y, Tryggvason K: Impaired endochondral ossification and angiogenesis in mice deficient in membrane-type matrix metalloproteinase I. Proc Natl Acad Sci USA 2000;97:4052-4057.

40 Jarvelainen H, Puolakkainen P, Pakkanen S, Brown EL, Hook M, Iozzo RV, Sage EH, Wight TN: A role for decorin in cutaneous wound healing and angiogenesis. Wound Repair Regen 2006;14:443-452.

41 Nelimarkka L, Salminen H, Kuopio T, Nikkari S, Ekfors T, Laine J, Pelliniemi L, Jarvelainen $\mathrm{H}$ : Decorin is produced by capillary endothelial cells in inflammation-associated angiogenesis. Am J Pathol 2001;158:345353. 